\title{
Modelagem de um Escritório de Gerenciamento de Processos: estudo em um campus de uma Universidade Federal Brasileira
}

\author{
Jamylle dos Santos Melo Lima ${ }^{I}$ \\ http://orcid.org/0000-0002-3837-5292
}

Victor Diogho Heuer de Carvalho ${ }^{\text {II }}$ http://orcid.org/0000-0003-2369-7317

Olival de Gusmão Freitas Júnior III http://orcid.org/0000-0003-4418-8386

I Universidade Federal de Alagoas, AL, Brasil. Graduada em Engenharia de Produção.

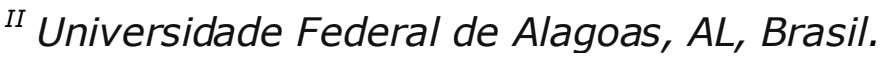

Professor dos Cursos de Engenharia de Produção e Civil do Campus do Sertão da Universidade Federal de Alagoas. Doutorando em Engenharia de Produção pela Universidade Federal de Pernambuco

III Universidade Federal de Alagoas, AL, Brasil.

Professor do Instituto de Computação da Universidade Federal de Alagoas. Doutor em Engenharia de Produção pela Universidade Federal de Santa Catarina.

http://dx.doi.org/10.1590/1981-5344/4071

As práticas de gestão organizacional precisam se adequar às exigências de eficiência e constante atualização tecnológica,não sendo diferente na Gestão Universitária, e a Gestão de Processos de Negócio contribui para que essa adequação seja alcançada.Este artigo destina-se a apresentar a modelagem de um Escritório de Gerenciamento de Processos (EGP)para um campus de uma universidade federal brasileira. Para isso, realizaramse coletas de dados por meio de um questionário com o 
gestor de um EGP externo, de uma entrevista estruturada com um gestor-chave interno e de análise de documentos da gestão universitária. Os sujeitos participantes foram escolhidos por conveniência e os dados foram analisados qualitativamente, de forma a se descreverem impressões, opiniões e históricos referentes ao tema central, e ainda para embasar o processo de modelagem do escritório, levando em consideração detalhes presentes nos documentos de gestão.Por fim, obtiveram-se os modelos dos processos que embasam o EGP utilizando a notação padrão para modelagem de processos de negócios, com apoio de um software especializado. Destaca-se que o processo de implantação de um EGP não é algo a ser executado a curto prazo, uma vez que envolve gestão de mudanças (na estrutura e na cultura organizacional) e diversas tramitações burocráticas.

Palavras-chave: Gestão de processos de negócio; Universidade federal brasileira; Gestão universitária; Escritório de gerenciamento de processos; Modelagem de processos de negócio.

\section{Modeling a Process Management Office: study on a campus of a Brazilian Federal University}

The practices of organizational management need to be adapted to the requirements of efficiency and constant technological updating, not being different in University Management, and the Business Process Management contributes to achieving this adequacy. This paper aims to present the modeling of a Process Management Office (PMO) for a campus of a Brazilian federal university. For this, data were collected through a questionnaire with the manager of an external PMO, a structured interview with a key internal manager and the analysis of documents of the university management. The subjects were chosen for convenience and the data were analyzed qualitatively, in order to describe impressions, opinions and histories 
referring to the central theme, and to base the process of modeling the office, considering details present in the management documents. Finally, the process models that support PMO using the standard notation for business process modeling were obtained with the support of a specialized software. It is noteworthy that the deployment process of an EGP is not something to be implemented in the short term, since it involves change management (in organizational structure and culture) and various bureaucratic formalities.

Keywords: Business process management; Brazilian federal university; University management; Process management office; Business process modeling.

Recebido em 09.08.2019 Aceito em 05.07.2021

\section{Introdução}

A Gestão de Processos de Negócio (Business Process Management, $B P M)$ surge como uma combinação de métodos unidos de apoio à gestão organizacional, para auxiliar na melhoria da qualidade de produtos e serviços, provendo gestão sistemática dos processos organizacionais e suas atividades (GABRYELCZYK;ROZTOCKI, 2018). Para obtenção dessas melhorias, algumas mudanças devem ser feitas, começando pelo planejamento estratégicos do uso dos diversos recursos empresariais, o que garante a formulação de uma estratégia adequada para gerir as mudanças necessárias para o uso de BPM (SENA;GUARNIERI, 2015).

Capote (2012) considera que a maioria delas mantém uma estratégia baseada em suposições funcionais e percepções desconectadas. Ele considera BPM como uma disciplina de gestão que busca promover a melhoria contínua, evidenciando a todo instante a devida importância que os processos de negócio possuem ao permanecerem alinhados, objetivando a entrega de valor para seus clientes.

No contexto da gestão das universidades públicas no Brasil, a gestão de processos tornou-se uma necessidade devido à complexidade das atividades acadêmicas, envolvendo ensino, pesquisa, gestão de recursos humanos, otimização do uso dos recursos financeiros públicos, uso de tecnologias da informação, além da orientação aos resultados (REINOSO;CASTILLO, 2019), de modo a apresentar à sociedade o impacto 
do que é produzido, assegurando que seja compreendida sua importância para o desenvolvimento da localidade na qual está inserida.

Conforme Lii e Li(2018), uma gestão universitária adequada é aquela em que a equipe responsável pela administração criou e consegue fazer uso apropriado de mecanismos para o processo decisório e para a gestão das operações envolvidas em cada área da universidade, garantindo que a alocação de recursos e os investimentos sejam realizados e otimizados por práticas pertinentes e eficazes. Seguindo essa tendência, a análise e modelagem de processos de negócios são ferramentas para garantir a qualidade nas atividades da universidade, garantindo que essas organizações possam se adaptar ao cenário de constantes mudanças em que as universidades modernas estão envoltas (SHUTOV, 2011).

$O$ presente artigo tem como objetivo relatar a modelagem de um escritório de gerenciamento de processos (EGP), por meio de seus processos centrais, para o Campus do Sertão da Universidade Federal de Alagoas (UFAL). Seu desenvolvimento baseou-se na aplicação de um questionário temático para coleta de informações de um EGP já implantando e funcional em outra instituição pública no estado de Alagoas, na realização de entrevista estruturada e na análise de documentos relacionados à gestão do campus, para garantir uma visão das percepções de um gestor-chave assim como para poder realizar a modelagem geral dos processos relacionados ao funcionamento do EGP proposto.

O restante do artigo está dividido da seguinte forma: a seção 2 apresenta um background sobre os principais tópicos envolvidos (Gestão Universitária e BPM); a seção 3 apresenta a metodologia aplicada para a pesquisa; a seção 4 apresenta a análise e discussão dos resultados obtidos; a seção 5 apresenta a modelagem do EGP; por fim, a seção 6 contém a conclusão do artigo.

\section{Background sobre a gestão universitárias e o uso do BPM}

Assim como qualquer tipo de gestão exercida em instituições públicas, a gestão das universidades públicas no Brasil deve ter como premissas o uso otimizado dos recursos públicos orçados, transparecendo à sociedade como esse uso está sendo feito para atender às suas necessidades (CATELLI;SANTOS, 2004). No caso das universidades, estas necessidades estão relacionadas ao desenvolvimento social, tecnológico e cultural das localidades onde estão situadas, através do tripé "ensino, pesquisa e extensão"(FREITAS JúNIOR et al., 2015).

Pressões sobre seu ambiente interno ocorrem uma vez que as universidades públicas, assim como qualquer outra organização pública, 
são sistemas abertos, facilmente influenciáveis pelo ambiente externo e seus stakeholders que desejam visualizar os resultados das atividades desenvolvidas (MARCHI et al., 2015). Para se adaptar ao cenário moderno de intensa cobrança social e constante necessidade de mudanças, a atualização do ambiente interno da universidade leva em consideração as dificuldades enfrentadas,as novas abordagens e estratégias que garantem que a universidade se contextualize frente às mudanças e supere essas dificuldades, tornando-se adequada conforme as exigências de seus ambientes interno e externo e os impactos das propostas e o grau de amadurecimento institucional, incluindo seus recursos humanos. (SAMPAIO; LANIADO, 2009).

Dentre as estratégias e abordagens adotadas pela gestão universitária para se manter atualizada e capaz de ser responsiva à sociedade, destacam-se o uso intensivo de Tecnologias da Informação e Comunicação e a adoção de métodos, técnicas, instrumentos/ferramentas gerenciais, com destaque para a gestão de processos (JESSE, 2010), e de mentalidade orientada ao empreendedorismo (CHEN, 2017). Destaca-se também a presença da Gestão do Conhecimento, uma vez que a necessidade de comunicar seus conhecimentos e compartilhar informações é inerente aos processos acadêmicos (CARLOS; CASTRO; SOUZA, 2018).

Surge então a necessidade de desenvolvimento de estratégias de BPM alinhadas com a estratégia maior das universidades públicas, uma vez que o uso de BPM promove nas organizações um melhor controle de seus processos de negócio por prover análise e monitoramento contínuos(KARABEGOVIC et al., 2018).

Rojas et al. (2011),Aurélio, Mendes e Bax (2018) e Silva et al.(2014) destacam que o BPM é uma ferramenta de mapeamento de fluxos de processos em organizações, funcionando como um meio de garantir a efetividade da gestão integrada, difundindo conhecimento e viabilizando o fluxo de informações úteis por toda a organização, para suportar as decisões e resoluções de problemas inerentes.

Para apoiar a gestão de processos na organização, fornecendo ferramentas e técnicas adequadas, os Escritórios de Gerenciamento de Processos (EGP) destacam-se como um staff transversal, uma vez que é capaz de atuar garantindo o desenvolvimento de BPM em todas as áreas da organização(BENEDICT et al., 2013).

Destacando as atividades executadas pelo EGP, uma vez que todas elas são práticas de BPM, pode-se mencionar(PAIM; CAULLIRAUX;CARDOSO, 2008): desenho/modelagem de processos, análise ambiental, definição de estratégia, asseguramento de patrocínio/financiamento, compreensão, seleção e priorização de processos, uso de técnicas de resolução de problemas, formação de 
grupos de diagnóstico de processos, definição de mudanças em processos, gestão de rotinas de processos, asseguramento da execução de processos, controle e monitoramento da execução de processos, fomento à inovação em processos, realização de benchmarking, condução da aprendizagem e da difusão do conhecimento sobre os processos, dentre outras atividades que podem ser derivadas.

A promoção do uso de ferramentas tecnológicas e de automação de processos, como sistemas de Gerenciamento de Processos de Negócio (Business Process Management Systems - BPMS), garante maior capacidade de adaptação às empresas frente ao cenário de mudanças constantes mencionado (WASZKOWSKI; NOWICKI;WORWA, 2018), facilitando a modelagem e o monitoramento por fazer uso da notação padrão de modelagem de processos de negócios (a BPMN, Business Process Model and Notation) (GEIGERet al., 2018).

Conclui-se este breve background com a reflexão de que o desenvolvimento tecnológico impulsionou o desenvolvimento da própria gestão de processos de negócios como destacado por Paim, Caulliraux e Cardoso (2008), desenvolvimento este promovido ora pela pesquisa acadêmica, ora pela pesquisa na iniciativa privada, demonstrando que a própria universidade que percebe a necessidade de uso de ferramentas avançadas de gestão pode ser a responsável pela sua criação. Desta forma, corrobora-se a relevância da temática abordada, já que a gestão universitária pode se tornar usuária das tecnologias desenvolvidas no âmago da instituição em que atua.

\section{Metodologia}

A pesquisa desenvolvida pode ser caracterizada como exploratória, de acordo com o ponto de vista dos objetivos, buscando proporcionar maior familiaridade com o problema, envolve um levantamento bibliográfico e de dados. Desta forma, a pesquisa envolve análise documental e estudo de caso que é a melhor estratégia quando o pesquisador tem pouco controle sobre o que se está sendo estudado(YIN, 2001). É ainda considerada qualitativa, pois a interpretação não requer a utilização de técnicas numéricas/estatísticas (MARCONI;LAKATOS, 2010), com análise indutiva de dados, tendo o EGP e os processos como foco principal. Utilizou-se amostragem não-probabilística com escolha por conveniência dos participantes,favorecendo significativamente à pesquisa por já conhecerem e terem experiência no assunto, fornecendo os dados necessários para assegurar que os resultados da pesquisa sejam alcançados(APPOLINÁRIO, 2008).

Preliminarmente às aplicações práticas da pesquisa desenvolvida, foi realizado um levantamento bibliográfico, de forma a apoiar o desenvolvimento teórico dos temas envolvidos, assim como para embasar 
a coleta de dados, de forma a identificar procedimentos e instrumentos adequados.

A aplicação prática foi dividida em três etapas, sendo necessária a utilização de diferentes instrumentos para a coleta de dados. A primeira etapa, realizada externamente à universidade, trata-se da análise do funcionamento de um EGP já implantado, onde foi submetido um questionário ao gestor responsável para coleta de dados sobre suas atividades.Na fase interna temos: a segunda etapa sendo a coleta de dados na UFAL, com entrevista à um gestor-chave e análise documental,sendo a última etapa a própria elaboração da proposta de modelo. O fluxo das atividades de pesquisa é apresentado na Figura 1.

Figura 1 - Fluxograma fundamental da etapa de coleta de dados.

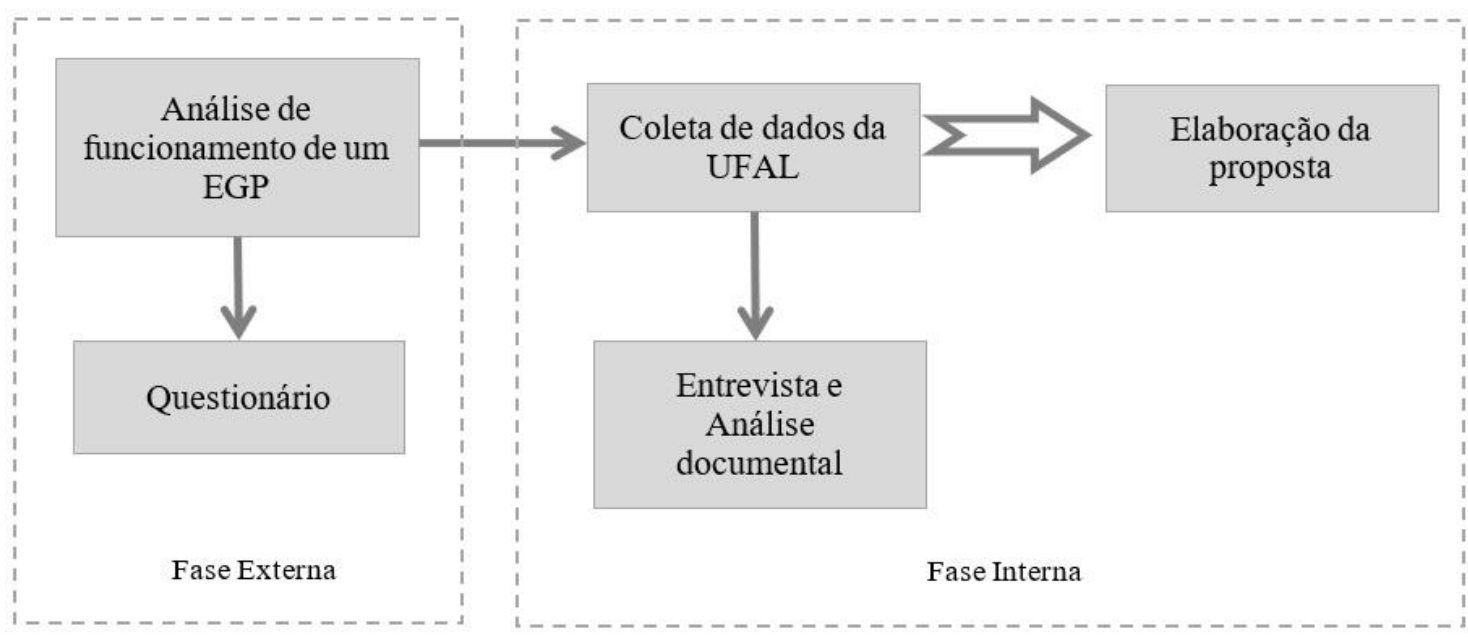

Fonte: os autores.

Na sequência, serão descritas cada uma das etapas metodológicas com maior detalhamento.

\subsection{Análise do funcionamento de um EGP}

O interesse em analisar uma organização que já tenha um escritório de processos implantado, é de obter contato direto com os responsáveis e coletar informações pertinentes ao processo de implantação desse escritório e seu real funcionamento (considerando que ele está de fato em plena atividade).Todo o material coletado nessa análise,destinou-se a auxiliar na formulação da proposta de modelo de EGP. Esta etapa, com suas atividades,está representada na Figura 2. 
Figura 2 - Análise de funcionamento de um EGP.

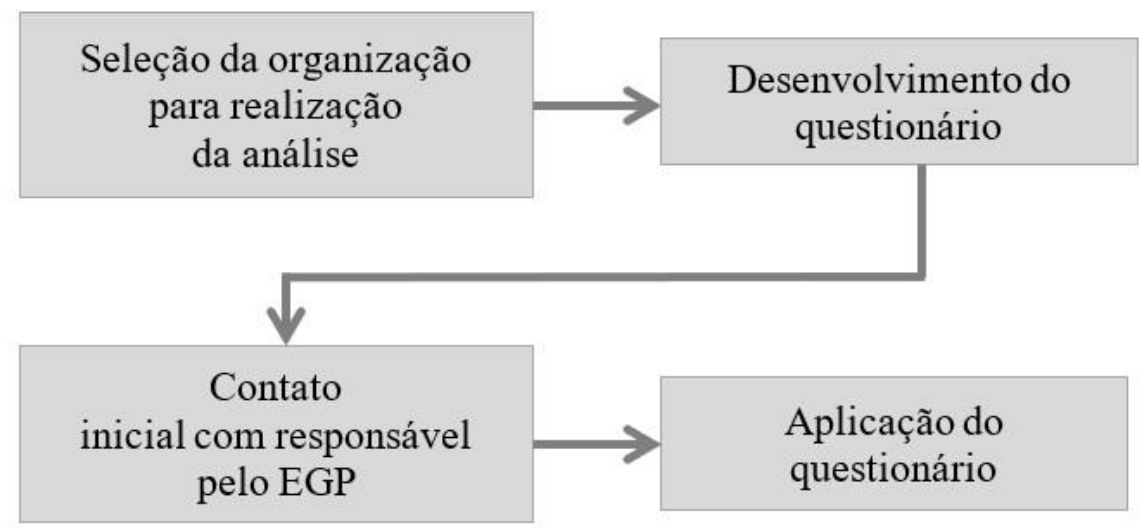

Fonte: os autores.

O primeiro desafio desta etapa foi definir a organização ideal para executar essa análise. Dentre as várias organizações brasileiras citadas como casos de sucesso na utilização das práticas de BPM e que possuem EGP implantado, o Ministério Público de Alagoas, localizado em Maceió, foi a organização selecionada. Essa decisão foi tomada por se tratar de uma das poucas organizações do estado de Alagoas, garantindo facilidade de acesso em um contexto de recursos escassos para locomoção e, além disso, por ser uma organização do setor público,o MP possui a demanda por eficiência na entrega de seus serviços à sociedade como um objetivo, assim como a UFAL.

Nesta etapa, a segunda decisão importante trata-se da definição de um instrumento adequado para realização da coleta de dados fundamentais para se alcançar o objetivo do estudo. De acordo com a rapidez e exatidão nas respostas, pelo favorecimento no horário de aplicação, dentre outros benefícios o questionário é um dos instrumentos mais utilizados para coleta de dados e foi o instrumento escolhido para execução desta etapa. O questionário foi obtido a partir da adaptação do questionário pré-existente de Macieira e Jesus (2013), de acordo com os principais aspectos a serem analisados no desenvolvimento de uma proposta de implantação de um EGP, que envolve inovação, investimentos, maturidade, qualificação e tecnologia.

Nessa adaptação, o questionário foi subdividido em seis etapas de análise, com questões abertas, fechadas e de múltipla escolha. A primeira etapa do questionário é formada por duas questões abertas para obtenção dos dados gerais do respondente. A segunda etapa é responsável pela identificação do histórico e status atual do gerenciamento de processos de negócio na organização, com seis questões. A terceira etapa busca compreender os resultados alcançados e os resultados esperados da organização com o gerenciamento de processos de negócio, com duas 
questões. A quarta etapa trata do entendimento das atividades que são realizadas pela organização de Gerenciamento de Processos de Negócio e o nível de maturidade de cada uma delas. A quinta etapa é a identificação das tecnologias e notações que a organização utiliza para execução das práticas da Gestão de Processos. A sexta e última etapa, trata das considerações finais, onde busca-se identificar a existência de restrições que atrapalhem a evolução do BPM na organização.

Diante do grande interesse que a organização teve em prestar informações para contribuir com o desenvolvimento do trabalho, uma data foi selecionada para aplicação do questionário. O questionário foi enviado via e-mail, e devolvido pelo mesmo canal.

\subsection{Coleta de dados na UFAL}

Para elaborar uma proposta de melhorias para uma organização é necessário ter informações relevantes a seu respeito, desde sua atuação em relação ao tema, até seu interesse nessa proposta. Portanto, a coleta de dados na UFAL (Figura 3), detalhada nesta etapa, foi também de total relevância para obtenção de um resultado satisfatório.

Figura 3 - Coleta de dados no Campus do Sertão da UFAL.

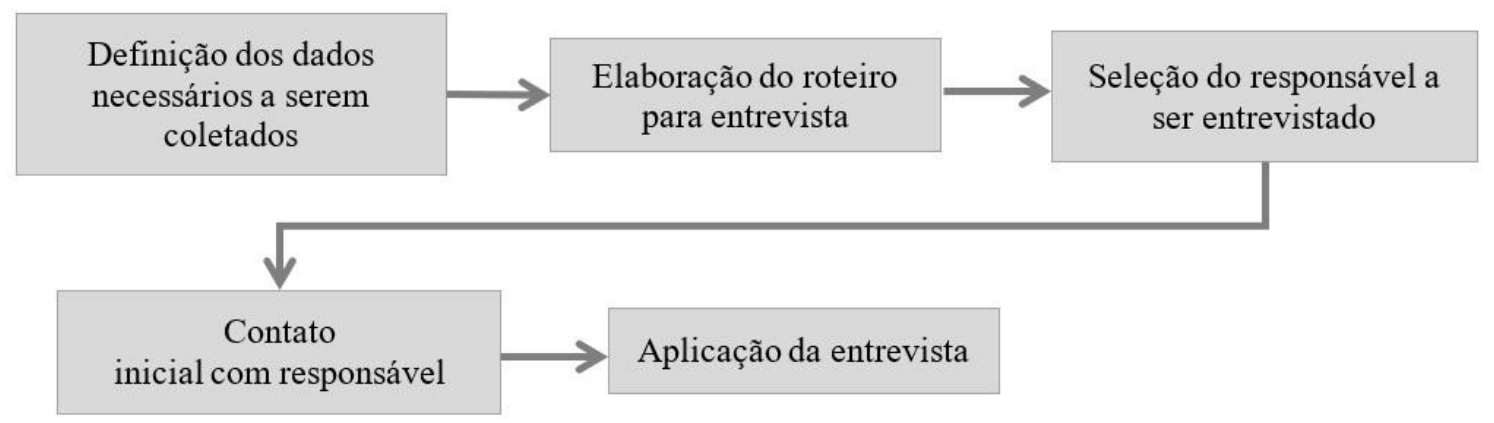

Fonte: os autores.

Inicialmente, foi feito um levantamento dos dados necessários a serem coletados, para possibilitar a elaboração de um roteiro de entrevista. Em outras palavras, foi definido o que poderia ser coletado para favorecer a elaboração final do modelo.

Feito esse levantamento, o roteiro da entrevista foi elaborado para investigar os seguintes aspectos: (i) estrutura organizacional do Campus Sertão (UFAL); (ii) posicionamento da organização em relação à busca por inovação; (iii) investimento em capacitação e tecnologia; (iv) resistência dos trabalhadores à mudança; (v) existência de profissional capacitado na área de Gestão de Processos; (vi) existência de práticas de BPM na organização; (vii) objetivo, missão e visão da organização; e (viii) considerações do responsável sobre a relevância do tema. 
A escolha do responsável a ser entrevistado foi feita de acordo com seu posicionamento na organização em relação à gestão. Devido a existência de um órgão dedicado à coordenação das atividades gerenciais no campus, seu coordenador foi o sujeito selecionado: primeiro ocorreu um contato informal para esclarecer a necessidade de uma entrevista e a apresentação dos objetivos do estudo;em seguida, uma reunião foi marcada para aplicação da entrevista.Além dos dados coletados na entrevista, foram analisados documentos de planejamento e a documentação existente sobre processos executados pela gestão do campus.

\subsection{Apoio à elaboração do modelo}

Dois outros trabalhos também foram utilizados como base para apoiar o desenvolvimento do modelo: "Estruturação de Escritório de Processos, no Centro de Ciências Exatas e Tecnologia da Universidade Federal do Estado do Rio de Janeiro - UNIRIO" de Pinho et al. (2008) e "Escritório de processos da Universidade Federal de Lavras: uma proposta de processos para seu funcionamento" de Balisa (2014).

$\mathrm{Na}$ etapa de desenho dos processos, a utilização do software Bizagi Process Modeler versão 3.0.0.014se faz necessária. Este software foi selecionado por ser um dos mais conhecidos e de uso difundido, pela praticidade de compreensão de sua interface e utilização da notação de modelagem utilizada, além de possuir uma versão gratuita com uma gama de funções que incluem, além da modelagem utilizando a notação padrão, opções de simulação conforme alguns processos estocásticos. O referido software também foi indicado pelo gestor que respondeu o questionário na etapa de análise do funcionamento de um EGP.

Documentos de descrição dos processos também foram desenvolvidos para uso pelos gestores como base para execução destes processos, contudo, estes documentos não serão apresentados neste artigo, por uma questão de limitação de espaço.

\section{Análise e discussão dos resultados}

Os resultados obtidos através da análise dos dados coletados nos dois momentos serão apresentados a seguir. Optou-se por não expor a identidade das pessoas chave participantes, por questões éticas.

\subsection{Ministério Público de Alagoas}

O Ministério Público de Alagoas vem trabalhando com BPM há aproximadamente três anos. O motivo que impulsionou a implantação da Gestão de Processos na instituição foi à visão do Procurador-Geral de Justiça em determinar a criação do Plano Estratégico Institucional com a intenção de tornar o MP uma instituição mais eficiente e eficaz, na 
resolutividade de suas atividades laborais internas/externas, culminando assim, em respostas mais céleres para sociedade.

O respondente afirma que inicialmente, os investimentos relativos à implantação do Escritório de Processos foram de baixo impacto financeiro, pois o setor foi criado no seio da Assessoria de Gestão Estratégica o que não gerou custos com a alocação de espaço físico. O fator que esclarece o baixo investimento para uma implantação com grande perspectiva de mudanças positivas, torna o projeto ainda mais interessante, pois diminui o impacto do risco de não alcançar as expectativas desejadas.

Para a organização, o BPM é visto como uma abordagem para melhoria, gestão do desempenho organizacional e redução de custos, além de ser uma maneira de documentar as rotinas de trabalho. O EGP está subordinado à assessoria ligada ao nível executivo e possui, formalmente, apenas um colaborador nessa área.

O responsável afirma que o nível de maturidade de BPM da organização ainda está em um estágio inicial e considera que muitas mudanças ainda devem ser feitas para que os resultados sejam ainda mais satisfatórios, ele descreve alguns dos principais resultados. Dentre eles estão: as melhorias no tempo de resposta de algumas atividades, a eliminação de gargalos, a redução de retrabalho e a eliminação de atividades que não agregam valor ao resultado produzido. $O$ que representa que os investimentos possuem retorno rápido e significativo, apesar do baixo envolvimento.

Os resultados esperados pela organização estão sendo alcançados à medida que o processo de sensibilização sobre BPM atinge os outros setores e unidades do MP. Isso permite que a instituição continue evoluindo para cada vez mais prestar um melhor serviço à sociedade. Outro ponto que merece destaque é a diminuição da dependência de outras pessoas-chave da organização para a execução das atividades.

A notação utilizada pela organização para a modelagem de processos é a BPMN, pois está alinhada ao Common Body of Knowledge(CBoK) de BPM(BENEDICT et al., 2013). Para tanto eles utilizam a ferramenta Bizagi por sua facilidade de uso e melhor aderência a notação padrão, além de ser uma ferramenta com versão gratuita disponível.

De acordo com o respondente, a principal restrição para a evolução do BPM na organização, trata-se da falta de orçamento e recursos disponíveis para esse tipo de investimento. Além disso, o mesmo considera que a resistência à mudança é uma grande barreira a ser enfrentada em busca da excelência.

Nota-se que as iniciativas de BPM não exigem altos investimentos iniciais dependendo da condição estrutural que for determinada para que a gestão atue. É possível adaptar as práticas de desenvolvimento da 
Gestão de Processos à realidade orçamentária da organização e ao quadro de funcionários existentes. Ou seja, apesar da falta de recursos para a ampliação destas práticas, é possível fazer a implantação de um Escritório de Gerenciamento de Processos em uma organização. Porém, assim como as práticas são limitadas, os resultados também serão.

\subsection{Universidade Federal de Alagoas}

Os resultados da entrevista com um gestor-chave na UFAL compõem uma outra base para a modelagem. Foi nesse momento da pesquisa que se pôde conhecer o funcionamento do Campus do Sertão da UFAL, suas limitações na entrega de seus serviços e as oportunidades que - EGP poderá trazer para a organização. Para melhor compreensão a respeito da organização em questão, inicialmente os dados coletados na entrevista resultam em uma análise do contexto organizacional a qual a UFAL pertence, identificando seu histórico, limitações, missão, princípios e valores, tudo isso levantado pelo entrevistado com apoio de documentação apresentada no ato.

A Universidade Federal de Alagoas (UFAL), maior instituição pública de Ensino Superior de Alagoas, foi fundada em 1961. Possui três campi:o campus A.C. Simões, com sede em Maceió e unidade em Rio Largo;o campus de Arapiraca, sediado em Arapiraca e com unidades em Viçosa, Penedo e Palmeira dos Índios; e o campus do Sertão, com sede em Delmiro Gouveia e unidade em Santana do Ipanema.

O Campus do Sertão, alvo da pesquisa realizada, foi inaugurado em 15 de março de 2010, e conta com aproximadamente 2.618 alunos distribuídos em 8 cursos.Possui cerca de140 servidores, dentre eles 39 técnicos-administrativos e 101 professores, além dos profissionais contratados como prestadores de serviço.

Através dos dados coletados na entrevista com o gestor-chave do Campus do Sertão, foi fácil identificar a estrutura organizacional: funcional por departamentos, o que pode ser considerado um ponto negativo para a organização, pois esse tipo de estrutura, onde sua atuação não possui orientação de processos transversais, possui uma abordagem com baixa capacidade de coordenação e principalmente com processos desconhecidos. Através dos manuais de políticas e procedimentos disponibilizados no momento da entrevista, foi possível verificar que iniciativas de BPM foram adotadas a partir de 2014. Esses manuais foram criados através do Sistema Integrado de Patrimônio, Administração e Contratos - SIPAC. Neles, há registros de fluxogramas de processos gerais da UFAL, contudo, não há atualizações desde o ano de lançamento.

O entrevistado reconhece a importância da implementação do BPM e de suas ferramentas, destacando a necessidade de um EGP para garantir maior maturidade na gestão de uma gama de atividades, cada uma delas 
dotada de complexidade, otimizando o gasto dos recursos públicos e os tempos despendidos para a resolução de problemas de gestão, assim como garantindo a integração e a melhoria das atividades administrativas.

\section{Modelagem de um EGP para o Campus do Sertão da UFAL}

De acordo com o que se pode levantar com a pesquisa bibliográfica, com a aplicação do questionário e com a entrevista realizada, o EGP deve ser considerado uma unidade de staff gerencial, tendo transversalidade de atuação entre todos os setores ou departamentos que necessitarem de seu apoio. Contudo, dentro da divisão de coordenações do campus estudado, há um grande alinhamento com a Direção Geral do Campus, com a Coordenação de Gestão Institucional (COGINST) e com o setor de Administração.Destaca-se que a COGINST é um órgão da gestão do campus capaz de coordenar as ações do EGP, uma vez que tem a visão geral, ao lado da Direção Geral, de todos os processos e suas atividades meio para garantir que as atividades fim (ensino, pesquisa e extensão) possam ser desempenhadas.

Seguindo esta tendência gerencial, o EGP tem como processos fundamentais, conforme Pinho et al. (2008): (i) seleção de processos, (ii) preparação das equipes para a gestão dos processos, (iii) gerenciamento da qualidade da gestão de processos, (iv) gerenciamento do portfólio de projetos da gestão de processos, ( $v$ ) gerenciamento dos próprios processos e (vi) definição de procedimentos para os processos.Conforme os dados levantados com a entrevista e a análise documental realizada, assim como levando em consideração os processos fundamentais para um EGP segundo Pinho et al. (2008), cada uma destas atividades pode ser modelada utilizando o software Bizagi conforme apresentado a seguir.

\subsection{Seleção de processos}

Visa identificar os processos que necessitam de ações de gerenciamento, ou seja, que podem ser melhorados. É considerado o principal processo do escritório, pois envolve a percepção de todos envolvidos na organização. A Figura 4 a seguir apresenta o modelo da seleção de processos. 
Figura 4 - Modelo de Seleção de Processos.

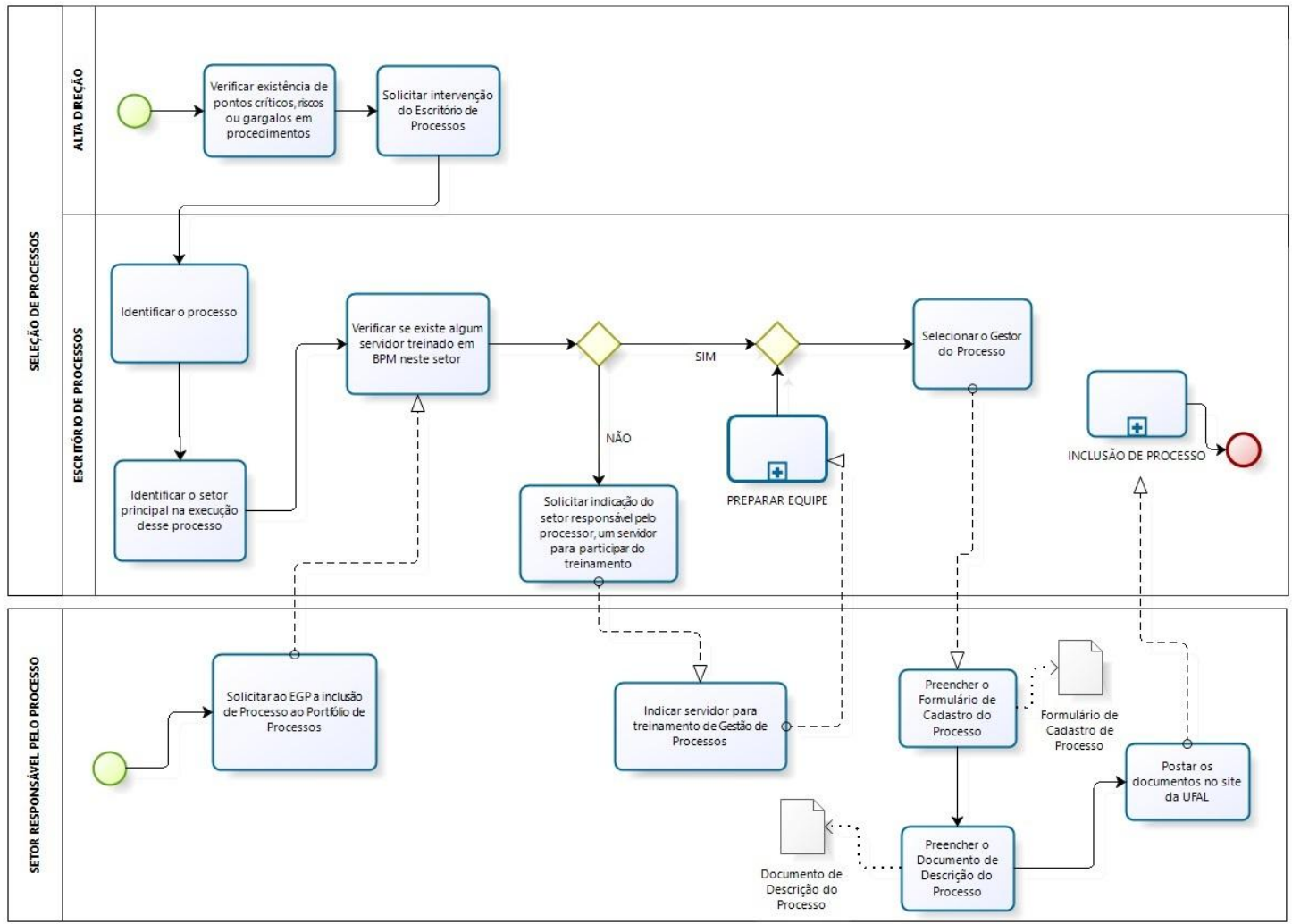

Fonte: os autores.

O processo de Seleção de Processos pode ser iniciado através de uma solicitação da alta direção através do reconhecimento de gargalos, pontos críticos ou riscos em algum processo. Além disso, se algum servidor de qualquer setor da organização reconhecer que existe uma falha no processo que executa, ele também pode solicitar ao EGP que analise 0 processo para que a inclusão do mesmo aconteça. Após identificar o processo e o setor a que ele se refere, o EGP deve solicitar a indicação do servidor que fará a modelagem do processo e que, portanto, necessitará de treinamento. Com a equipe treinada o processo segue em sua fase de preenchimento do Documento de Descrição do Processo e do Formulário de Cadastro de Processo. E por fim, os documentos são arquivados e inicia-se o subprocesso de Inclusão de Processo.

\subsection{Preparação de equipes de gestão de processos}

Fundamental na dissipação do conhecimento sobre Gestão de Processos na organização. Este processo dará condição aos servidores para que executem a modelagem dos processos e mantenham seu 
funcionamento otimizado. A Figura 5 representa a modelagem de preparação de equipes.

Figura 5 - Modelo de Preparação de Equipes para Gestão de Processos.

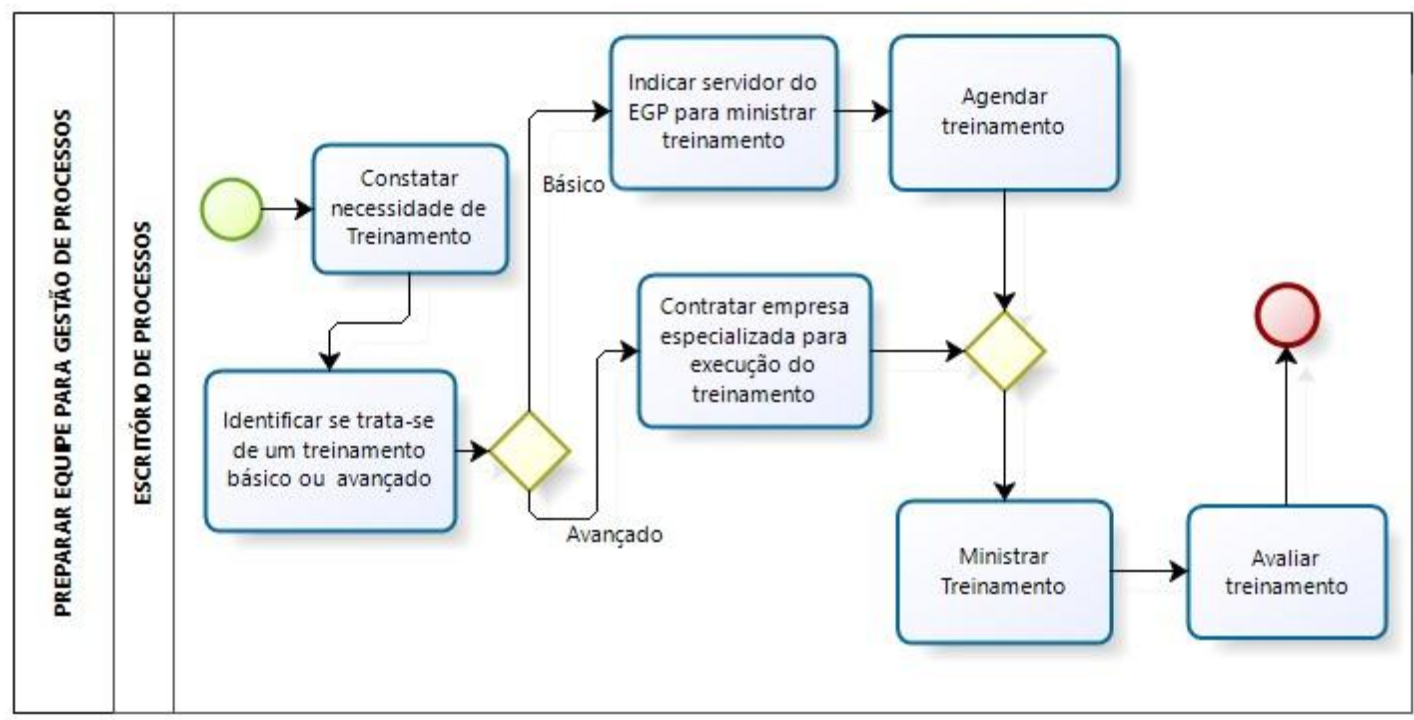

Fonte: os autores.

O processo inicia-se com a constatação da necessidade do treinamento, que deve ser definido como básico, ou avançado. No caso de um treinamento básico, deve ser indicado um servidor do EGP para ministrar o treinamento, que logo em seguida é agendado, executado e por fim avaliado. Já no caso da necessidade de um treinamento avançado, deve ser contratada pelas vias formais da universidade uma empresa para executar o treinamento, que por fim, também será avaliado.

\subsection{Gerenciamento da qualidade da gestão de processos}

Destina-se à normatização dos padrões de execução das atividades e a garantia de que a gestão de processos funciona da melhor maneira possível. A Figura 6 representa o modelo de gerenciamento da qualidade. 
Figura 6 -Modelo de Gerenciamento da Qualidade da Gestão de Processos.

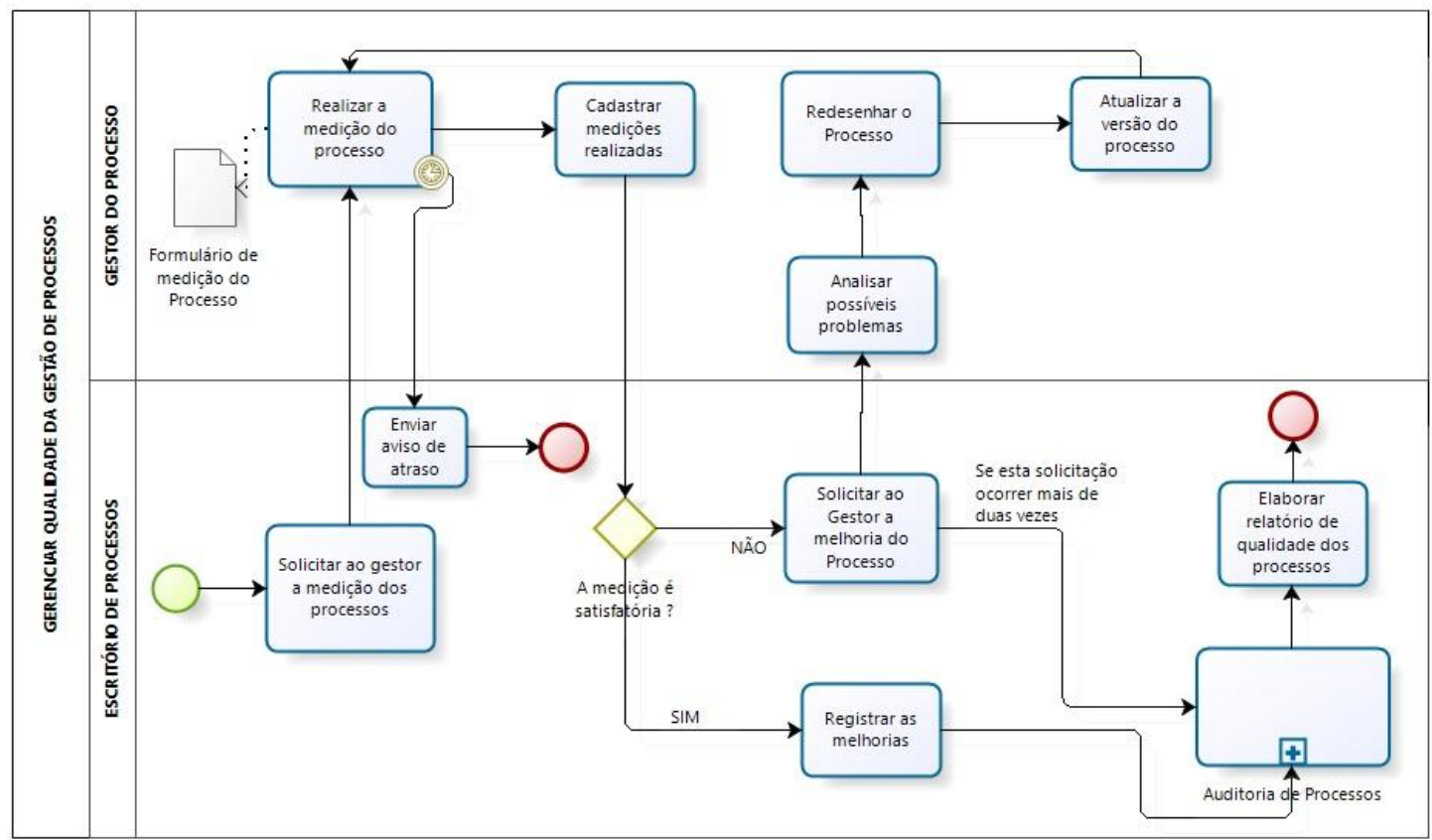

Fonte: os autores.

Inicialmente no processo de Gerenciamento da Qualidade da Gestão de Processos, o EGP solicita ao Gestor do Processo que faça a medição do processo, que ocorre através do preenchimento do Formulário de Medição do Processo. A medição deve ocorrer através das métricas estabelecidas nos indicadores-chave de desempenho.

Para essa atividade, o escritório de processos deverá designar um tempo para que a mesma seja executada, caso o tempo seja ultrapassado, o gestor de processos recebe uma notificação de atraso. Em sequência a medição realizada deve ser cadastrada, se ela for satisfatória - EGP registra as melhorias e abre o processo de auditoria. Caso a medição não seja satisfatória o gestor do processo é solicitado para realizar as melhorias, analisando os possíveis problemas e redesenhando o processo.

Após o redesenho, a nova versão do processo deve ser registrada, retomando assim a fase inicial deste processo que é a medição. Caso seja solicitado mais de duas vezes correção de um processo, deve ser iniciado o processo de auditoria. Por fim, após o processo de auditoria, deve ser elaborado um relatório de qualidade, informando a real situação do processo. 


\subsection{Gerenciamento do portfólio de projetos da gestão de processos}

Tem como objetivo organizar os processos de acordo com o macroprocesso que ela pertence e revisá-los periodicamente para que seja enviado ao processo de Gerenciamento da Qualidade da Gestão de Processos. A Figura 7 apresenta o modelo proposto para o EGP da UFAL.

Figura 7 - Modelo de Gerenciamento do Portfólio de Projetos de Gestão de Processos.

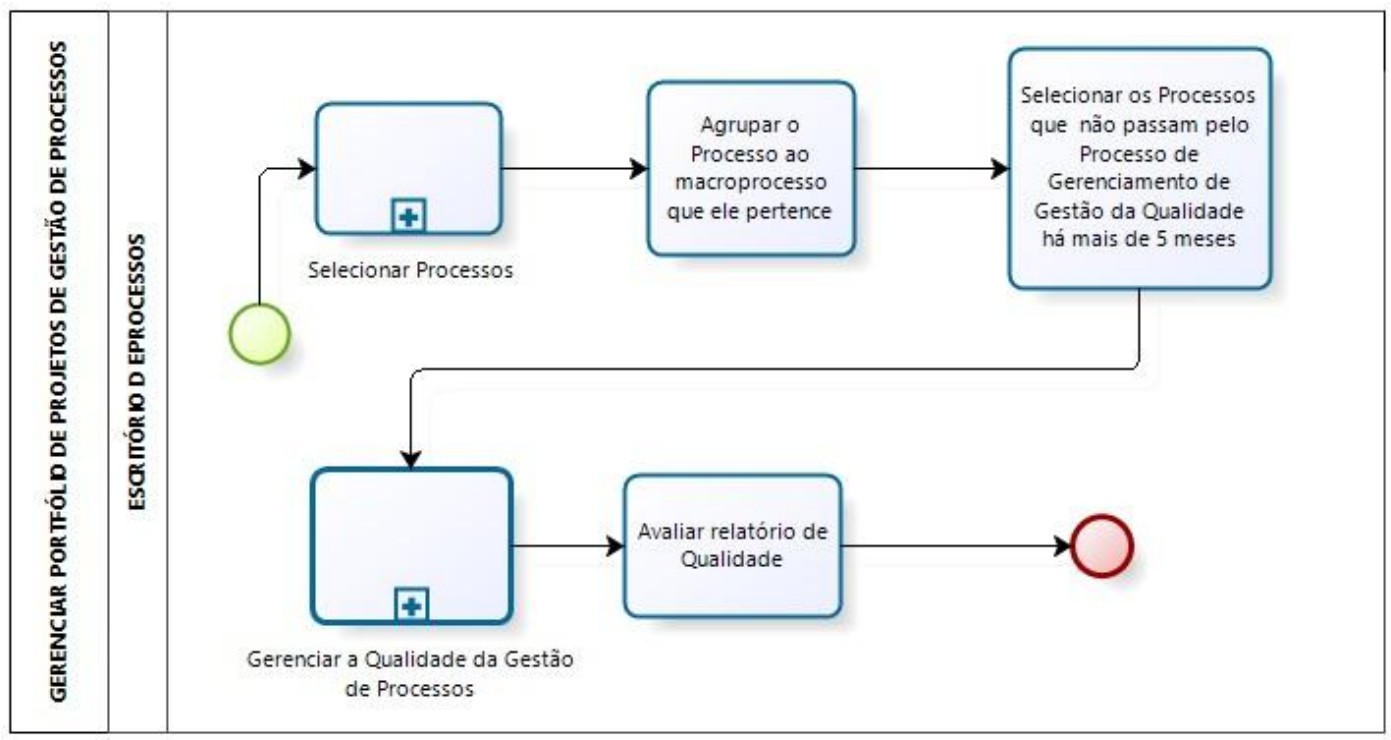

Fonte: os autores.

O processo de Gerenciamento do Portfólio de Projetos de Gestão de Processos tem uma ligação direta com o processo de seleção, pois é onde este processo é iniciado. Após a seleção de processos, os processos são agrupados de acordo com o macroprocesso a que ele pertence. Como método de investigação e garantia da qualidade dos processos existentes no portfólio, os processos são frequentemente analisados e a cada 5 meses são enviados ao processo de Gerenciamento da Qualidade de Gestão de Processos. Para finalizar este processo, o relatório de qualidade deve ser analisado.

\subsection{Gerenciamento dos próprios processos (gerenciamento interno)}

O escritório de processos tem como um de seus objetivos, mostrar a importância do Gerenciamento dos Processos, portanto, o EGP é tido como um modelo para toda organização e seu funcionamento é o que torna a gestão de processos evolutiva. A Figura 8 representa o modelo deste processo. 
Figura 8 -Modelo de Gerenciamento dos Processos Internos do Escritório.

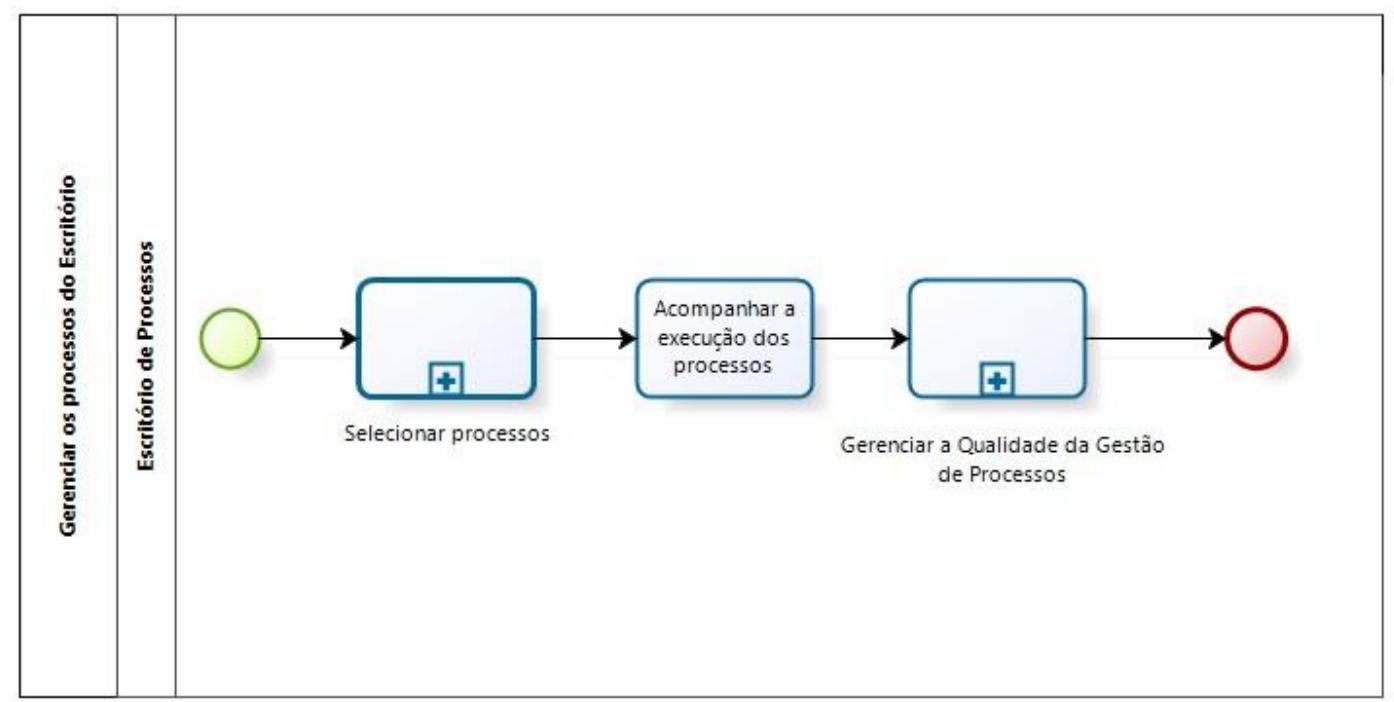

Fonte: os autores.

Este processo é iniciado através do processo de "Seleção de Processos", que neste caso é tido como um subprocesso realizável. Após a execução da seleção, é responsabilidade do escritório acompanhar a execução dos processos e por fim, encaminhado para o processo de "Gerenciamento da Qualidade de Gestão de Processos", que neste caso, também é considerado um subprocesso realizável.

\subsection{Definição dos procedimentos para os processos}

Tem como objetivo a criação de manuais que detalhem os procedimentos de cada processo, enriquecendo 0 portfólio $e$ compartilhando com todos os servidores da organização a melhor forma de executar determinado processo. A Figura 9 representa este processo ponta-a-ponta. 
Figura 9 -Modelo de Definição de Procedimentos para os Processos.

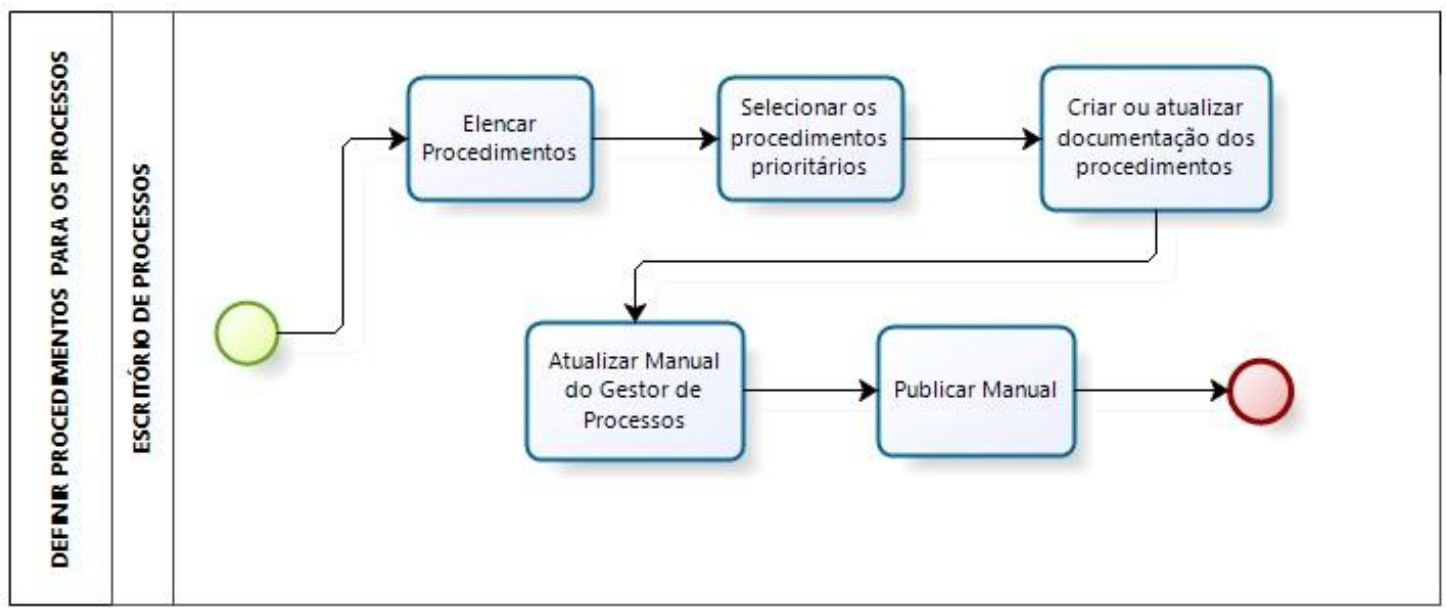

Fonte: os autores.

Os processos são elencados e selecionados de acordo com sua prioridade e em seguida os documentos dos procedimentos são criados, ou atualizados no caso de já existirem. Em seguida, o manual do Gestor de Processo deve ser atualizado e publicado na página da Organização.

\section{Conclusões}

O desenvolvimento do presente estudo possibilitou a modelagem de uma série de processos relacionados ao EGP proposto para o Campus do Sertão (UFAL). Para que isso pudesse ser realizado, antes foram coletados dados sobre o EGP externo à instituição além de se realizar uma entrevista estruturada com um gestor-chave e uma análise de documentos de gestão. Essa análise tornou possível a certificação do quanto a Gestão de Processos pode ajudar no desenvolvimento dessas organizações, as adequando as cobranças do mercado e tornando possível o crescimento delas.

A proposta de modelo, apresentada aqui de forma sintética, descreveu procedimentos, apresentou os requisitos necessários e o fluxo a ser seguido para sua implantação, sendo uma solução facilitadora para os processos de gestão do campus e sanando estrategicamente outro problema da organização. A implantação de um EGP na UFAL não é um projeto a ser executado à curto prazo, pois envolve fatores como a mudança na estrutura e cultura organizacional, além de questões burocráticas que necessitam da aprovação da direção geral e dos conselhos universitários. A paciência, o comprometimento e a 
comunicação entre os servidores, são fundamentais para o sucesso da iniciativa.

Por se tratar de uma proposta, a pesquisa deixa em aberto diversos temas futuros a serem abordados, pois, com a aceitação e aplicação do que foi proposto, diferentes indicadores de desempenho podem ser aplicados para identificar como as tarefas estão sendo realizadas, medir o desempenho das práticas aplicadas, verificar se a organização está atingindo seus objetivos e até mesmo dando continuidade a modelagem dos processos, verificando também a maturidade que se conseguiu alcançar com o EGP.

\section{Referências}

APPOLINÁRIO, F. Metodologia Científica: filosofia e prática da pesquisa. São Paulo:Cengage Learning, 2009.

AURÉLIO, M.; MENDES, S.; BAX, M. P. BPM and ECM: Similarities, differences, conceptual, and technological limits,Transinformação, v. 30, n.1, p. 95-105, 2018. Disponível em: https://doi.org/10.1590/231808892018000100008. Acesso em: 13 nov. 2018.

BALISA, M. A. Escritório de processos da Universidade Federal de Lavras: uma proposta de processos para seu funcionamento. Monografia, Universidade Federal de Lavras, Lavras, MG, Brasil, 2014. Disponível em: http://www.bsi.ufla.br/wp-content/uploads/2014/09/Marcelo-AdaltonBalisa TCC-Marcelo-Balisa-200920432 1578.pdf. Acesso em: 30 out. 2018.

BENEDICT, T.; MOORE, C.; BILODEAU, N.; VITKUS, P. BPM CBOK: version 3.0. 1. ed. Association of Business Process Management Professionals, 2013.

CAPOTE, G. BPM para todos: uma visão geral abrangente, objetiva e esclarecedora sobre gerenciamento de processos de negócio.1. ed. Rio de Janeiro: Gart Capote, 2012.

CARLOS, L.; CASTRO, E.; SOUZA, L. A Utilização das Práticas de Gestão do Conhecimento e Facilitadores Estratégicos em uma Instituição de Ensino Superior, Revista Gestão Universitária na América Latina, v. 11, n. 2, p.319-341, 2018.Disponível em: https://doi.org/10.5007/19834535.2018v11n2p319. Acesso em: 13 nov. 2018.

CATELLI, A.; SANTOS, E. S. Mensurando a criação de valor na gestão pública. Revista de Administração Pública, v. 38, n.3, p. 423-449, 2004. Disponível em: 
http://bibliotecadigital.fgv.br/ojs/index.php/rap/article/view/6544/5128. Acesso em: 10 nov. 2018.

CHEN, Z. A study on the practice path of university management. In: International Conference on Information, Communication and Engineering,Xiamen, 2017. Anais eletrônicos [...] Xiamen: IEEE, 2017. p. 322-324.Disponível em: https://doi.org/10.1109/ICICE.2017.8479021. Acesso em: 30 out. 2018.

FREITAS JÚNIOR, O.G.; BARROS, P. A. M.; BARBIRATO, J. C. C.; BRAGA, M. M.; DE CARVALHO, V. D. H. Reestruturando o Modelo de Universidade Pública Brasileira para Atender aos Novos Desafios Gerenciais. In:XV Colóquio Internacional de Gestão Universitária, Mar del Plata, 2015. Anais eletrônicos [...] Mar del Plata: Instituto de Pesquisas e Estudos em Administração Universitária, 2015. p. 1-15. Disponível em: https://repositorio.ufsc.br/xmlui/handle/123456789/136255. Acesso em: 10 nov. 2018.

GABRYELCZYK, R.; ROZTOCKI, N. Business process management success framework for transition economies. Information Systems Management, v. 35, n.3, p. 234-253, 2018. Disponível em:https://doi.org/10.1080/10580530.2018.1477299. Acesso em: 13 nov. 2018.

GEIGER, M.; HARRER, S.; LENHARD, J.; WIRTZ, G. BPMN 2.0: The state of support and implementation. Future Generation Computer Systems, v. 80, p. 250-262, 2018. Disponível em:

https://doi.org/10.1016/j.future.2017.01.006. Acsso em: 10 nov. 2018.

JESSE, N. Business Process Automation for Universities: Enabling Effective and Efficient University Management. In: International Conference on Management and Service Science, Wuhan, 2010. Anais eletrônicos [...] Wuhan: IEEE, 2010, p. 1-4.Disponível em:

https://doi.org/10.1109/ICMSS.2010.5576875. Acesso em: 30 out. 2018.

KARABEGOVIC, A.; BUZA, E.; OMANOVIC, S.; KAHROVIC, A. Adoption of BPM systems for process design in a higher education institution. In: 41st International Convention on Information and Communication Technology, Electronics and Microelectronics, Opatija, 2018. Anais eletrônicos [...] Opatija: IEEE, 2018., p. 552-557.Disponível em:

https://doi.org/10.23919/MIPRO.2018.8400105. Acesso em: 30 out. 2018.

LII, P.; LI, S. Contemporary University Institutional Governance from Service Quality Perspectives. 2018 In: 15th International Conference on Service Systems and Service Management, Hangzhou, 2018. Anais 
eletrônicos [...] Hangzhou: IEEE, 2018, p. 1-14. Disponível em: https://doi.org/10.1109/ICSSSM.2018.8465065. Acesso em: 30 out. 2018.

MACIEIRA, A.; JESUS, L. Pesquisa Nacional em Gerenciamento de Processos de Negócio. 1. ed., BPM Global Trends, 2013.

MARCONI, M. A. ; LAKATOS, E. M. Técnicas de Pesquisa. 7. ed., São Paulo: Atlas, 2010.

PAIM, R.; CAULLIRAUX, H. M.; CARDOSO, R. Process management tasks: A conceptual and practical view. Business Process Management Journal, v. 14, n. 5, p. 694-723, 2008. Disponível em:

https://doi.org/10.1108/14637150810903066. Acesso em: 17 nov. 2018.

PINHO, B.; CAPPELLI, C.; BAIÃO, F.; SANTORO, F.; PAIM, R. ; NUNES, V. Estruturação de Escritório de Processos. Relatórios Técnicos do DIA/UNIRIO, v. 1, Rio de Janeiro: UNIRIO, 2008. Disponível em: www.seer.unirio.br/index.php/monografiasppgi/article/download/216/221. Acesso em: 13 nov. 2018.

REINOSO, J. R. R. ; CASTILLO, D. C. C. Importance of ICT's Use in Business Management and Its Contribution to the Improvement of University Processes. In:BOTTO-TOBAR, M.; BARBA-MAGGI, L.; GONZÁLEZ-HUERTA, J.; VILLACRÉS-CEVALLOS, P.; GÓMEZ , O. S.; UVIDIA-FASSLER , M. I. (Eds.).Information and Communication Technologies of Ecuador, Cham: Springer International Publishing, p. 241-252, 2019. Disponível em: https://doi.org/10.1007/978-3-03002828-2 18. Acesso em: 10 nov. 2018.

ROJAS, R. S. B.; JULIATTO, D. L. J. ; FACCHINI, É.; PEREIRA, R. P. Utilização da metodologia BPM para adequação de um sistema de gestão integrada e retenção de conhecimento em uma instituição pública de ensino superior. Revista Gestão Universitária na América Latina, Edição Especial, p. 115-132, 2011. Disponível em: https://doi.org/10.5007/1983-4535.2011v4nespp115. Acesso em: 13 nov. 2018.

MARCHI, A.; ROSSETTO, C. R.; LIMA, C. M.; PEREIRA, D. G. O Processo de Mudança em uma Instituição Pública sobre a Ótica das Capacidades Dinâmicas, Revista Gestão Universitária na América Latina,v. 11, n. 2, p. 175-197, 2018. Disponível em: https//doi.org/10.5007/19834535.2018v11n2p175. Acesso em: 13 nov. 2018.

SAMPAIO, R. M.; LANIADO, R. N. Uma experiência de mudança da gestão universitária: o percurso ambivalente entre proposições e realizações. $R A P$ 
Revista de Administração Pública, v. 43, n. 1, p. 151-174, 2009. Disponível em: https://doi.org/10.1590/S0034-76122009000100008. Acesso em: 10 nov. 2018.

SENA, A. S.; GUARNIERI, P. Enterprise Resource Planning governamental: a percepção dos servidores atuantes no Projeto Ciclo do Ministério da Justiça quanto à implementação. Revista de Administração Pública, v. 49, n.1, p. 207-230, 2015. Disponível em: https://doi.org/10.1590/003476121408. Acesso em: 10 nov. 2018.

SHUTOV, A. Reengineering University: Modeling Business Processes to Achieve Strategic Goals. In: WRYCZA, S. (Ed.). Research in Systems Analysis and Design: Models and Methods. Berlin, Heidelberg: Springer Berlin Heidelberg, 2011, p. 3-14. Disponível em:

https://doi.org/10.1007/978-3-642-25676-9 1. Acesso em: 17 nov. 2018.

SILVA, L. C. ; POLETO, T. ; DE CARVALHO, V. D. H. ; COSTA, A. P. C. S. Selection of a business process management system: An analysis based on a multicriteria problem. In: IEEE International Conference on Systems, Man and Cybernetics, 2014, San Diego. Anais eletrônicos [...] San Diego: IEEE, 2014. Disponível em:https://doi.org/10.1109/SMC.2014.6973923. Acesso em: 14 nov. 2018.

WASZKOWSKI, R.; NOWICKI, T.; WORWA, K. Corporate efficiency improvement with business process automation. In: 22nd International Conference on Circuits, Systems, Communications and Computers, 210, 2018, MATEC Web Conf. Anais eletrônicos [...] MATEC Web Conf., 2018.Disponível em: https://doi.org/10.1051/matecconf/201821002012. Acesso em: 17 nov. 2018.

YIN, R. K. Estudo de caso: planejamento e métodos. 2. ed., Porto Alegre: Bookman., 2001. 